\title{
A review of rare complications of maxillary sinus floor augmentation
}

\author{
Sung Woon On ${ }^{1,3,4}$, Seoung-Won Cho ${ }^{2,3,4}$, Byoung-Eun Yang ${ }^{2,3,4}$ \\ ${ }^{I}$ Division of Oral and Maxillofacial Surgery, Hallym University Dongtan Sacred Heart Hospital, Hwaseong, \\ ${ }^{2}$ Division of Oral and Maxillofacial Surgery, Hallym University Sacred Heart Hospital, Anyang, \\ ${ }^{3}$ Graduated School of Clinical Dentistry, Hallym University, Chuncheon, \\ ${ }_{4}^{4}$ Institute of Clinical Dentistry, Hallym University, Chuncheon, Korea
}

\begin{abstract}
J Korean Assoc Oral Maxillofac Surg 2019;45:351-356)
Maxillary sinus floor augmentation (MSFA) is an essential procedure for implant installation in the posterior maxillary area with vertical alveolar bone deficiency. For the past several decades, MSFA has been refined in terms of surgical methods along with technical progress, accumulation of clinical studies, and development of graft materials and surgical instruments. Although some complications in MSFA are inevitable in clinical situations, management of those complications in MSFA has been well established thanks to many clinicians and researchers. Nevertheless, some rare complications may arise and can result in fatal results. Therefore, clinicians should be well aware of such rare situations and complications associated with MSFA. In this review, the authors present several rare complications regarding MSFA, along with corresponding management strategies through a thorough review of the literature.
\end{abstract}

Key words: Maxillary sinus floor augmentation, Sinus floor augmentation, Sinus graft, Complication

[paper submitted 2019. 11. 15 / revised 2019. 11. 25 / accepted 2019. 11. 25]

\section{Introduction}

Maxillary sinus floor augmentation (MSFA) was first introduced by Tatum ${ }^{1}$, and its first official discussion occurred in a publication by Boyne and James². Since then, MSFA has been refined in terms of surgical methods along with technical progress, accumulation of clinical studies, and development of graft materials and surgical instruments. At present, MSFA is an essential procedure for implant installation in the posterior maxillary area with vertical alveolar bone deficiency. There are two approaches to perform MSFA, a lateral approach and a transcrestal approach. Both are safe and effective procedures to produce adequate bone formation for

\footnotetext{
Byoung-Eun Yang

Division of Oral and Maxillofacial Surgery, Hallym University Sacred Heart Hospital, 22 Gwanpyeong-ro 170beon-gil, Dongan-gu, Anyang 14068, Korea

TEL: +82-31-380-3870 FAX: +82-31-380-1726

E-mail:face@hallym.ac.kr

ORCID: https://orcid.org/0000-0002-4446-6772

(c) This is an open-access article distributed under the terms of the Creative Commons Attribution Non-Commercial License (http://creativecommons.org/ licenses/by-nc/4.0/), which permits unrestricted non-commercial use, distribution, and reproduction in any medium, provided the original work is properly cited.

Copyright (C) 2019 The Korean Association of Oral and Maxillofacial Surgeons. All rights reserved.
}

implant installation in the pneumatized maxillary sinus.

Furthermore, both approaches show a high success rate in graft and implant survival ${ }^{3,4}$. However, some intraoperative and postoperative complications can result from MSFA. These complications include perforation of the Schneiderian membrane, bleeding, displacement of graft material, sinus congestion, maxillary sinusitis, infection, and others ${ }^{5,6}$. Although complications in MSFA are inevitable in clinical situations, management of these complications in MSFA has been well established thanks to many clinicians and researchers. Nevertheless, some rare complications may arise, for which clinicians may be unprepared, resulting in difficulty and postponement of treatment. In some cases, such situations can lead to fatal problems. Therefore, clinicians should be well aware of rare situations and complications associated with MSFA. This review aims to present several rare complications related to MSFA to enable clinicians to cope with such circumstances appropriately. 


\section{Rare Complications}

\section{Migration of dental implant to another site}

Displacement of a dental implant into the maxillary sinus is relatively common, and its possible causes include low primary stability of dental implant relative to bone, insufficient experience of the surgeon, pressure from the temporary denture, inadequate manipulation of non-integrated implant, and others $^{7}$. In cases of migration, two treatment modalities have been suggested for removal of the displaced dental implants, formation of a bony window on the maxillary anterolateral surface via an intraoral approach and functional endoscopic sinus surgery (FESS) via a transnasal approach. These modalities can be performed selectively upon considering the existence of an oroantral fistula or an obstructed ostium of the maxillary sinus. An intraoral approach can remove displaced implants and close an oroantral fistula using local flaps. However, it cannot provide sufficient management of an obstructed ostium of the maxillary sinus. Although FESS cannot close an oroantral fistula, it allows simultaneous management of an ostium of the maxillary sinus, treatment of paranasal sinus infection, and removal of dental implants ${ }^{8}$.

There are some possible locations into which dental implants can be displaced. Theoretically, the implants can be displaced into the paranasal sinus connected with the maxillary sinus. However, other than the maxillary sinus, only cases of implant displacement into the sphenoid and ethmoid sinuses have been reported ${ }^{9-11}$. Haben et al. ${ }^{9}$ first reported a case with a migrated dental implant into the ethmoid sinus. However, the patient did not undergo MSFA. In this case, although the authors tried to remove the displaced implant using an endoscopic approach, they could not find the implant, and the procedure was terminated. Fortunately, there was no foreign body detected upon postoperative plain films. Bakhshalian et al. ${ }^{10}$ also reported migration of a dental implant into the ethmoid sinus. A 60-year-old male patient who underwent MSFA via a transcrestal approach showed one implant displaced into the ethmoid sinus adjacent to the orbit on computed tomography (CT) scan. The displaced dental implant was removed by formation of a bony window via an intraoral approach, and an endoscopic camera was used to visualize the migrated implant. The authors presented a hypothesis for this migration as ciliary movement of the columnar epithelium in the maxillary sinus for drainage.

To the best of our knowledge, there is only one case report discussing an implant displaced into the sphenoid sinus. Fe- lisati et al. ${ }^{11}$ demonstrated that a 45 -year-old female patient with a dental implant displaced into the maxillary sinus during a surgical procedure showed spontaneous migration of the implant into the sphenoid sinus two weeks after surgery in the posterior maxillary area. The implant was removed using an endoscope via a transnasal approach, and the patient showed an uneventful postoperative recovery. The authors presumed that the reason for the displacement was presence of a large-diameter maxillary sinus ostium, and the tip of the implant was engaged with the spheno-ethmoidal recess due to the physiological response of mucociliary clearance.

Another infrequent case example is that of an implant that has been displaced into the anterior cranial fossa. Cascone et al. ${ }^{12}$ reported a case showing implant displacement in the anterior cranial fossa. A 50-year-old male complained of persistent rhinorrhea after installation of a dental implant in the left maxilla. He also showed a hematoma on the left medial canthus. A CT scan showed the implant displaced into the anterior cranial fossa between the medial orbital wall and the sphenoid bone. The implant was removed endoscopically via the left nasal cavity. Since the implant was in contact with the cerebral parenchyma, dural repair was also performed using bovine pericardium product. The patient showed no specific complications during follow-up up to one year after surgery.

Based on the cases above, we conclude that prevention of the migration is the best way to avoid negative patient outcome when performing an implant installation in the post maxillary area. Since implants that had been displaced into the ethmoid sinus, sphenoid sinus, and anterior cranial fossa all migrated from the maxillary sinus, clinicians should identify major causes of displacement into the maxillary sinus and carefully perform implant installation in the posterior maxillary area, especially when performing MSFA. In addition, if the implant migrates into the maxillary sinus during installation, it should be immediately removed, or the patient should be referred to oral and maxillofacial surgeons in general hospitals for the immediate management.

\section{Infections associated with MSFA}

Infection after MSFA is common, with an incidence up to $21 \%$ reported in the literature ${ }^{13}$. Contamination of the maxillary sinus by bacteria during MSFA seems to be inevitable. Postoperative infection following MSFA can occur in two ways, infection of graft material below the elevated sinus membrane and infection of the sinus itself ${ }^{14}$. The former occurs much more frequently than the latter ${ }^{15}$. The reported 
symptoms of sinus graft infection are severe pain, fistula formation, recurrent facial swelling at postoperative two to three weeks, abscess, fever, and loss of graft materials ${ }^{16}$. The common symptoms of sinus infection consist of pain, swelling, tenderness, pus discharge, and purulent exudate from the nasal cavity ${ }^{17}$. Most infections after MSFA are bacterial and can be effectively treated with antibiotics. However, in a case of a major sinus graft infection, further management including endoscopic sinus exploration, surgical exploration, and rinsing is required, in addition to administration of systemic antibiotics $^{13,18}$. When performing MSFA, most authors prescribe antibiotics and anti-inflammatory or corticosteroid with $0.2 \%$ chlorhexidine mouthwash preoperatively and postoperatively to prevent infection ${ }^{5,13,16,19}$

Fungal infection after MSFA rarely occurs, but Sohn et al. ${ }^{20}$ presented an unusual case of Aspergillus infection associated with MSFA. In their case report, a 48-year-old male had undergone MSFA in the right maxillary sinus in a private dental clinic. Three dental implants were placed in the right maxilla. After six months, however, those implants were neither covered with soft tissue nor stable. As a result, one implant was removed by his dentist, and the patient was referred to a university hospital for further evaluation and management. CT scan showed a floating grafted bone from the sinus floor that was encapsulated by a thickened Schneiderian membrane. After surgical exposure of the right maxillary sinus, the infected graft materials were removed and sent for histopathologic examination, whereby Aspergillosis was detected.

The authors of this case reported that the grafted material was infected when the implant was placed, or the sinus already had been infected by the fungus prior to implant surgery. Although the specific source of fungus infection in this patient was not apparent, the authors believed that smoking and perforation of the sinus membrane during MSFA might be contributing factors.

Methicillin-resistant Staphylococcus aureus (MRSA) infection associated with MSFA was reported as a rare case. Ward et al. ${ }^{21}$ presented an 82-year-old female with MRSA sinusitis following MSFA and subsequent placement of 11 endoosseous implants. The patient's medical history consisted of hypertension, immunocompetency, depression, hypothyroidism, and osteoarthritis. She had undergone multiple medical treatments and surgical procedures to manage chronic sinusitis over 20 years. After implant installation, she complained of persistent and worsening symptoms of sinusitis and underwent surgery, including endoscopic bilateral ethmoidectomy, maxillary antrostomy, sphenoidotomy, and exploration of the frontal sinus by an otolaryngologist. Two years after the operation, the patient showed left frontal swelling and pus discharge from a bilateral maxillary antrostomy area. Pus culture confirmed the presence of MRSA; therefore, intravenous administration of vancomycin and levofloxacin was provided, and frontal and maxillary sinusotomy was performed. A large amount of purulent debris around multiple dental implants protruding into the sinus was observed during surgery.

Therefore, the patient was referred to the department of oral and maxillofacial surgery for evaluation of the possibility of implant removal. After surgical removal of all 11 implants, she showed uneventful healing with six weeks of intravenous administration of antibiotics. It was assumed that sinus disease was a preexisting condition and might have been responsible for this problematic situation. Therefore, Ward et al. ${ }^{21}$ proposed that patients with a previous history of chronic sinus problems should be evaluated thoroughly before MSFA.

Another uncommon infection occurring with MSFA is maxillary sinusitis associated with peri-implantitis (MSPI). Recently, Park et al. ${ }^{22}$ demonstrated the treatment outcome of eight patients diagnosed with MSPI after MSFA. In their case series, diagnosis of MSPI was based on clinical symptoms and findings obtained via cone-beam CT (CBCT), which showed mucosal thickening and obstruction of the ostium. The most frequent clinical symptom was nasal obstruction, followed by mucoid rhinorrhea and loss of smell. In addition, all the dental implant prostheses showed idiosyncratic screw loosening at the time of diagnosis. All eight patients were treated with implant removal and administration of antibiotics, with or without the modified Caldwell-Luc operation (CLOP). All clinical symptoms in the eight patients were resolved at the six-month postoperative follow-up. The authors in this case series suggested with caution that the lateral approach may be a better method than the transcrestal approach to prevent MSPI when the residual alveolar ridge height is at a borderline level since the eight patients consisted of six who had undergone the transcrestal approach and two who had undergone the lateral approach. Conclusively, the authors suggested that progression of peri-implantitis in an augmented sinus floor site may cause maxillary sinusitis, and early intervention for MSPI is important. Just as periapical lesions of natural teeth can cause odontogenic maxillary sinusitis, it is thought the peri-implantitis may also cause maxillary sinusitis. Therefore, we should be aware of this possibility.

Through the cases above, we assume that a thorough preoperative history is important when patients show clinical symptoms associated with maxillary sinusitis, and an imme- 
diate intervention such as medication is needed. Furthermore, clinicians should carefully consider surgical treatment if the symptoms do not improve with medication.

\section{Benign paroxysmal positional vertigo}

Benign paroxysmal positional vertigo (BPPV) is one of the complications associated with the transcrestal approach using osteotomes for MSFA. Although BPPV is not uncommon, its importance is worth mentioning in this review.

Dix and Hallpike ${ }^{23}$ first introduced the term 'BPPV' and suggested the Dix-Hallpike test for confirmation. The incidence of BPPV associated with the transcrestal approach is less than $3 \%$ in the literature ${ }^{24}$. The typical symptoms of BPPV are short, strong vertigo and nausea caused by angular positional change of the head. These symptoms usually subside within several days to weeks; however, some cases show persistent symptoms.

BPPV associated with the transcrestal approach is considered a secondary occurrence. Secondary BPPV is often triggered by head and neck trauma and surgeries such as stapedectomy, cochlear implantation, MSFA using osteotomes, and molar extraction. The hypothesis of origination of secondary BPPV is that surgical trauma, especially pressure generated by procedures such as MSFA using osteotomes, can cause detachment of the otolith, and the detached otolith may move into the posterior semicircular canal because of patient hyperextended and tilted head position during surgery.

Some treatment modalities have been recommended for management of BPPV, including medication, surgery, and vestibular exercises. First, we should be well aware of the primary management techniques for the desired repositioning of the otolith into the utricle. This maneuver, called the canalith repositioning procedure, was introduced by Epley ${ }^{25}$ in 1992. The procedure begins with seating the patient in a dental unit chair and tilting his or her head 45 degrees to the affected side. Then, the patient lies down, and the headrest is tilted to angle the patient's head backward while maintaining the turned head posture. Next, the patient's head is slowly rotated to the unaffected side. Finally, the patient slowly returns to a vertical sitting position. After this procedure, the following instructions are recommended: the patient should not lie back, bend over, or tilt the head for two days; the patient should not turn the head to the affected side during sleep. This technique is very useful to alleviate the symptoms of BPPV. Therefore, if a patient complains of dizziness after MSFA using osteotomes, this procedure should be considered for management of BPPV. If nausea persists, use of medication for motion sickness can be considered, although it does not generally work ${ }^{26}$.

According to Vernamonte et al. ${ }^{27}$, oral and maxillofacial surgeons should recognize and respond to such a situation. Furthermore, the immediate referral of the patient to the otolaryngologist should be considered for early diagnosis and treatment. This approach will provide relief to the patient and maintain a medico-legal guarantee for surgeons, in addition to reducing the risk of long-lasting BPPV symptoms. It is suggested that risk of postoperative BPPV be explained to patients who need MSFA using osteotomes. If BPPV occurs, oral and maxillofacial surgeons should manage it wisely using the methods described above.

\section{Devitalization of adjacent teeth following MSFA}

There has been a concern that devitalization of adjacent teeth may occur as a rare complication of MSFA. Theoretically, when the Schneiderian membrane is lifted, the blood supply to the apices of adjacent teeth may be jeopardized due to rupture of blood vessels. As a result, there is a possibility of vitality loss or pulp necrosis of the teeth. Romanos et al. ${ }^{28}$ reported a case series about the loss of pulp vitality after MSFA. In their case series, three patients showed devitalization of neighboring teeth following MSFA, and they underwent endodontic treatment performed by endodontists. The preoperative evaluation presented no dental caries and intact pulp vitality determined by cold testing. Conventional MSFA was performed for all patients with two-stage implant installation. Two of the three patients were asymptomatic, and the other showed periapical radiolucency. There was no evidence of occlusal trauma, bruxism, or parafunctional habits in the preoperative clinical exam. Therefore, the authors in this case series concluded that pulp necrosis occurred when the Schneiderian membrane was elevated over the apices of the teeth, which are close to the sinus floor.

In 2018, a retrospective radiographic study about this topic was published. Beck et al. ${ }^{29}$ conducted a study on 257 patients who met the inclusion criteria and assessed the probability of tooth devitalization retrospectively after MSFA, with a value less than $0.7 \%$. Only one case showed tooth devitalization a few months after implant placement. In this case, augmented graft materials were extended over the root of the tooth on the postoperative panoramic view. Although the exact mechanism or cause was not identified, this complication seems to be very rare. The authors in this retrospective 
study assumed that the extremely low probability of devitalization of adjacent teeth after MSFA might be attributed to the existence of collateral circulation in the alveolar bone of the maxilla. However, the authors also supposed the possibility of asymptomatic teeth with devitalized pulp. Therefore, they mentioned that a prospective study should be performed to investigate this topic further.

CLOP is a more invasive surgery of the sinus compared to MSFA and has a very low probability of tooth devitalization (i.e., two of 464 case $^{30}$ ). Accordingly, it seems logical that MSFA, which is a less invasive surgery than CLOP, has an extremely low frequency of devitalization of adjacent teeth after surgery. Although this complication is not common, we should be aware of its potential and be able to conduct appropriate clinical and radiological examinations for evaluation when in doubt.

\section{Conclusion}

MSFA is a necessary procedure in daily clinical practice, and management of related common complications has been established. Nevertheless, unexpected rare complications ay occur. Therefore, MSFA should be performed after a thorough preoperative examination, precise radiological evaluation and analysis, and appropriate patient selection. In the event of a sudden medical situation resulting from rare complications of MSFA, it may be challenging to deal with the problem. Hence, oral and maxillofacial surgeons should be aware of these complications and their preventive measures, as well as the solutions.

\section{ORCID}

Sung Woon On, https://orcid.org/0000-0001-6192-1530

Seoung-Won Cho, https://orcid.org/0000-0002-3955-5623

Byoung-Eun Yang, https://orcid.org/0000-0002-4446-6772

\section{Authors' Contributions}

S.W.O. made the conception of this review, and drafted the manuscript. S.W.C. revised the manuscript, and helped to draft the manuscript. B.E.Y made the conception of this review, revised the manuscript, and made a final approval of the manuscript. All authors have read and approved the final manuscript.

\section{Conflict of Interest}

No potential conflict of interest relevant to this article was reported.

\section{References}

1. Smiler DG, Johnson PW, Lozada JL, Misch C, Rosenlicht JL, Tatum $\mathrm{OH} \mathrm{Jr}$, et al. Sinus lift grafts and endosseous implants. Treatment of the atrophic posterior maxilla. Dent Clin North Am 1992;36:151-86; discussion 87-8.

2. Boyne PJ, James RA. Grafting of the maxillary sinus floor with autogenous marrow and bone. J Oral Surg 1980;38:613-6.

3. Pjetursson BE, Tan WC, Zwahlen M, Lang NP. A systematic review of the success of sinus floor elevation and survival of implants inserted in combination with sinus floor elevation. J Clin Periodontol 2008;35(8 Suppl):216-40.

4. Tan WC, Lang NP, Zwahlen M, Pjetursson BE. A systematic review of the success of sinus floor elevation and survival of implants inserted in combination with sinus floor elevation. Part II: transalveolar technique. J Clin Periodontol 2008;35(8 Suppl):241-54.

5. Schwartz-Arad D, Herzberg R, Dolev E. The prevalence of surgical complications of the sinus graft procedure and their impact on implant survival. J Periodontol 2004;75:511-6.

6. van den Bergh JP, ten Bruggenkate CM, Disch FJ, Tuinzing DB. Anatomical aspects of sinus floor elevations. Clin Oral Implants Res 2000;11:256-65.

7. Kluppel LE, Santos SE, Olate S, Freire Filho FW, Moreira RW, de Moraes M. Implant migration into maxillary sinus: description of two asymptomatic cases. Oral Maxillofac Surg 2010;14:63-6.

8. Chiapasco M, Felisati G, Maccari A, Borloni R, Gatti F, Di Leo F. The management of complications following displacement of oral implants in the paranasal sinuses: a multicenter clinical report and proposed treatment protocols. Int J Oral Maxillofac Surg 2009;38:1273-8.

9. Haben CM, Balys R, Frenkiel S. Dental implant migration into the ethmoid sinus. J Otolaryngol 2003;32:342-4.

10. Bakhshalian N, Sim YC, Nowzari H, Cha HS, Ahn KM. Accidental migration of a dental implant into the ethmoid sinus following a transalveolar sinus elevation procedure. Clin Implant Dent Relat Res 2015;17:360-4.

11. Felisati G, Lozza P, Chiapasco M, Borloni R. Endoscopic removal of an unusual foreign body in the sphenoid sinus: an oral implant. Clin Oral Implants Res 2007;18:776-80.

12. Cascone P, Ungari C, Filiaci F, Gabriele G, Ramieri V. A dental implant in the anterior cranial fossae. Int J Oral Maxillofac Surg 2010;39:92-3.

13. Barone A, Santini S, Sbordone L, Crespi R, Covani U. A clinical study of the outcomes and complications associated with maxillary sinus augmentation. Int J Oral Maxillofac Implants 2006;21:81-5.

14. Carreño Carreño J, Gómez-Moreno G, Aguilar-Salvatierra A, Martínez Corriá R, Menéndez López-Mateos ML, Menéndez-Núñez M. The antibiotic of choice determined by antibiogram in maxillary sinus elevation surgery: a clinical study. Clin Oral Implants Res 2018;29:1070-6.

15. Testori T, Drago L, Wallace SS, Capelli M, Galli F, Zuffetti F, et al. Prevention and treatment of postoperative infections after sinus elevation surgery: clinical consensus and recommendations. Int $\mathrm{J}$ Dent 2012;2012:365809.

16. Urban IA, Nagursky H, Church C, Lozada JL. Incidence, diagnosis, and treatment of sinus graft infection after sinus floor elevation: a clinical study. Int J Oral Maxillofac Implants 2012;27:449-57.

17. Kim J, Jang H. A review of complications of maxillary sinus augmentation and available treatment methods. J Korean Assoc Oral 
Maxillofac Surg 2019;45:220-4.

18. Kahnberg KE, Vannas-Löfqvist L. Sinus lift procedure using a 2-stage surgical technique: I. Clinical and radiographic report up to 5 years. Int J Oral Maxillofac Implants 2008;23:876-84.

19. Felice P, Scarano A, Pistilli R, Checchi L, Piattelli M, Pellegrino G, et al. A comparison of two techniques to augment maxillary sinuses using the lateral window approach: rigid synthetic resorbable barriers versus anorganic bovine bone. Five-month post-loading clinical and histological results of a pilot randomised controlled clinical trial. Eur J Oral Implantol 2009;2:293-306.

20. Sohn DS, Lee JK, Shin HI, Choi BJ, An KM. Fungal infection as a complication of sinus bone grafting and implants: a case report. Oral Surg Oral Med Oral Pathol Oral Radiol Endod 2009;107:37580.

21. Ward BB, Terrell JE, Collins JK. Methicillin-resistant Staphylococcus aureus sinusitis associated with sinus lift bone grafting and dental implants: a case report. J Oral Maxillofac Surg 2008;66:2314.

22. Park WB, Han JY, Oh SL. Maxillary sinusitis associated with periimplantitis at sinus floor augmented sites: case series. Implant Dent 2019;28:484-9.

23. Dix MR, Hallpike CS. The pathology, symptomatology and diagnosis of certain common disorders of the vestibular system. Ann Otol Rhinol Laryngol 1952;61:987-1016.

24. Di Girolamo M, Napolitano B, Arullani CA, Bruno E, Di Girolamo S. Paroxysmal positional vertigo as a complication of osteotome sinus floor elevation. Eur Arch Otorhinolaryngol 2005;262:631-3.
25. Epley JM. The canalith repositioning procedure: for treatment of benign paroxysmal positional vertigo. Otolaryngol Head Neck Surg 1992;107:399-404.

26. Saker M, Ogle O. Benign paroxysmal positional vertigo subsequent to sinus lift via closed technique. J Oral Maxillofac Surg 2005;63:1385-7.

27. Vernamonte S, Mauro V, Vernamonte S, Messina AM. An unusual complication of osteotome sinus floor elevation: benign paroxysmal positional vertigo. Int J Oral Maxillofac Surg 2011;40:216-8.

28. Romanos GE, Papadimitriou DE, Hoyo MJ, Caton JG. Loss of pulp vitality after maxillary sinus augmentation: a surgical and endodontic approach. J Periodontol 2014;85:43-9.

29. Beck F, Lauterbrunner N, Lettner S, Stavropoulos A, Ulm C, Bertl K. Devitalization of adjacent teeth following maxillary sinus floor augmentation: a retrospective radiographic study. Clin Implant Dent Relat Res 2018;20:763-9.

30. DeFreitas J, Lucente FE. The Caldwell-Luc procedure: institutional review of 670 cases: 1975-1985. Laryngoscope 1988;98:1297-300.

How to cite this article: On SW, Cho SW, Yang BE. A review of rare complications of maxillary sinus floor augmentation. J Korean Assoc Oral Maxillofac Surg 2019;45:351-356. https://doi. org/10.5125/jkaoms.2019.45.6.351 